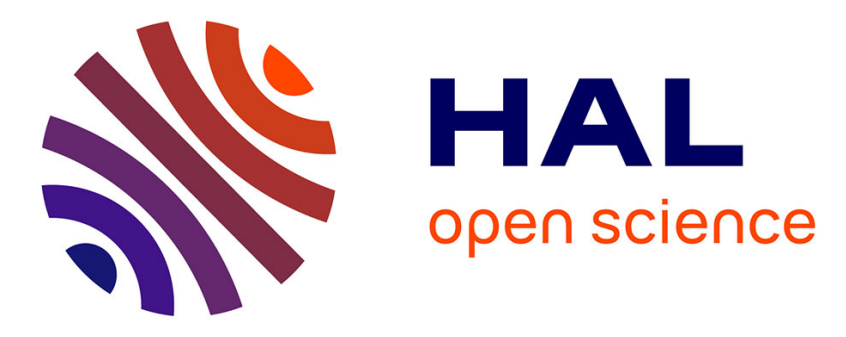

\title{
Why Intuitionistic Relevant Logic Cannot Be a Core Logic
}

Joseph Vidal-Rosset

\section{To cite this version:}

Joseph Vidal-Rosset. Why Intuitionistic Relevant Logic Cannot Be a Core Logic. Notre Dame Journal of Formal Logic, 2017, 58 (2), pp.241-248. 10.1215/00294527-3839326 . hal-01469864

\section{HAL Id: hal-01469864 https://hal.science/hal-01469864}

Submitted on 7 Jun 2017

HAL is a multi-disciplinary open access archive for the deposit and dissemination of scientific research documents, whether they are published or not. The documents may come from teaching and research institutions in France or abroad, or from public or private research centers.
L'archive ouverte pluridisciplinaire HAL, est destinée au dépôt et à la diffusion de documents scientifiques de niveau recherche, publiés ou non, émanant des établissements d'enseignement et de recherche français ou étrangers, des laboratoires publics ou privés. 
Notre Dame Journal of Formal Logic

Advance publication

\title{
Why Intuitionistic Relevant Logic Cannot Be a Core Logic
}

\author{
Joseph Vidal-Rosset
}

\begin{abstract}
At the end of the 1980s, Tennant invented a logical system that he called "intuitionistic relevant logic" (IR, for short). Now he calls this same system "Core logic." In Section 1, by reference to the rules of natural deduction for IR, I explain why IR is a relevant logic in a subtle way. Sections 2, 3, and 4 give three reasons to assert that IR cannot be a core logic.
\end{abstract}

\section{IR in a Nutshell}

1.1 Motivation of IR Intuitionistic relevant logic (IR, for short) is a logical system invented by Tennant at the end of the 1980s to prove all theorems of Heyting logic without accepting the intuitionistic absurdity rule $\perp E$ (i.e., $\frac{\perp}{B} \perp E$ ).

Tennant claims that, in his logical system, neither the "first Lewis paradox," the sequent provable in intuitionistic logic by application of the intuitionistic absurdity rule $\perp E$, that is, ex contradictione quodlibet,

$$
\neg A, A \vdash_{i} B,
$$

nor the negate-conclusion version of the first Lewis paradox, that is, the following sequent provable in minimal logic,

$$
\neg A, A \vdash_{m} \neg B,
$$

are provable in IR. The subtle goal of IR can be understood thanks to Tennant's slogan:

If one needs a slogan to help with orientation, ours is a method of relevantizing "at the level of the turnstile." As a result, there will be some tweaking of the rules for the logical operators (in the natural deduction or sequent setting); but these tweakings are not as to change their established meanings. [7, p. 711]

Received January 26, 2014; accepted July 30, 2014

$\diamond$ Final volume, issue, and page numbers to be assigned. 2010 Mathematics Subject Classification: 03B20, 03B47 Keywords: intuitionistic logic, relevant logic, minimal logic (C) 2017 by University of Notre Dame $\quad 10.1215 / 00294527-3839326$ 
1.2 Tweaking the rules with IR Before seeing how IR tweaks the rules of intuitionistic logic, note the unchanged rules of minimal logic belonging also to IR:

$$
\frac{\neg A \quad A}{\perp} \perp E \quad \frac{A \quad B}{A \wedge B} \wedge I \quad \frac{A}{A \vee B} \vee I \frac{B}{A \vee B} \vee I
$$

Now let us see the "tweaking" that Tennant (see [4], [6], [7]) has made. In the group of introduction rules, there is an additional rule $\rightarrow I_{i r}$. (Labels $\rightarrow I_{i r}$ and $\vee E_{i r}$ are mine.) The other changes are notifications of obligatory or permissible discharge:

$$
\begin{array}{lcc}
\hline A(i) & \frac{A}{A} \square(i) & A \diamond(i) \\
\vdots & \vdots & \vdots \\
\frac{\perp}{\neg A} \neg I,(i) & \frac{\perp}{A \rightarrow B} \rightarrow I_{i r},(i) & \frac{B}{A \rightarrow B} \rightarrow I,(i)
\end{array}
$$

The symbol $\square$ means that the discharge of $A$ is obligatory. If you can deduce a contradiction from an open assumption $A$, then either you can conclude $\neg A$ by application of rule $\neg I$, or you can introduce a conditional with $A$ as the antecedent and any well-formed formula as the consequent, like $B$; but in both cases you have to discharge $A$. In the other case, when you can deduce a formula $B$ from $A$, if $B$ is not a contradiction, then the discharge of $A$ is $\diamond$ (i.e., just permissible). I must specify now a crucial metarule of IR.

Definition 1.1 (Proof in IR) A deduction $\mathscr{D}$ is a proof in IR if and only if only IR-rules of inference are used in $\mathscr{D}$ and if and only if $\mathscr{D}$ is in normal form. According to Prawitz [3, p. 34], a proof is in normal form "if no formula occurrence is both the consequence of an application of an I-rule and major premise of an application of an E-rule." A proof is said to be in normal form if it is redex-free, that is, if no introduction rule for a connective is followed immediately by an elimination rule of the same connective. If a deduction is not in normal form, then it is not a proof in IR.

1.2.1 Why (ECQ) is claimed to be unprovable in IR At this stage, we do not need to know more about the rules of IR to understand why Tennant claims that (ECQ $\neg$ ) and (ECQ) are not among the deducibilities of IR, while, for example, the formula expressing the ex falso quodlibet in the axioms of Heyting logic, that is,

$$
\vdash_{i} \neg A \rightarrow(A \rightarrow B),
$$

is nevertheless also the theorem of IR.

\section{Proof}

$$
\frac{\frac{\frac{\neg A}{1}^{\frac{\perp}{A}}}{\frac{A \rightarrow B}{\neg}} I_{i r}, 2}{\neg A \rightarrow(A \rightarrow B)} \rightarrow I, 1
$$

Note that this proof of (1) does not prove (ECQ) and that it is impossible to use a conditional elimination rule in order to deduce (ECQ): such a proof would be an abnormal proof, that is, a nonproof in IR (see Definition 1.1). 
1.2.2 Why (ECQ $\neg)$ is claimed to be unprovable in $\mathbf{I R}$ To prevent a proof of (ECQ $\neg)$ in IR, vacuous discharge (or weakening) is banned from IR when the base of assumptions is reducible to contradiction. Indeed, suppose that there is no restriction on the use of vacuous discharge, as in minimal logic and in intuitionistic logic; then the following deduction schema

$$
\frac{\perp}{B \rightarrow \perp} \rightarrow I
$$

is a sufficient proof of (ECQ $\neg$ ) because, by the intuitionistic definition of negation, the formula $B \rightarrow \perp$ is $\neg B$. But one must add that, if vacuous discharge is replaced by the following rule of weakening, ${ }^{1}$ defined by David, Nour, and Raffalli [1, p. 38],

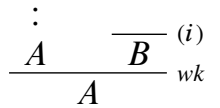

then this deduction schema shows that (ECQ $\neg$ ) is always provable:

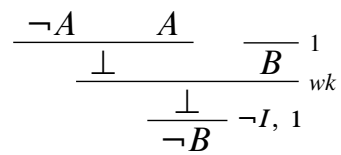

To avoid the provability in IR, one must therefore ban this instance of weakening: ${ }^{2}$

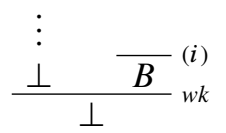

1.2.3 Tweaking of elimination rules and acceptance of disjunctive syllogism For computational reasons explained in Autologic [4], Tennant replaces the usual rules $\wedge E$ and $\rightarrow E$ by these "parallelized" rules:

$$
\begin{aligned}
& \square(i) \underbrace{A, B} \square(i) \\
& \bar{B} \square(i)
\end{aligned}
$$

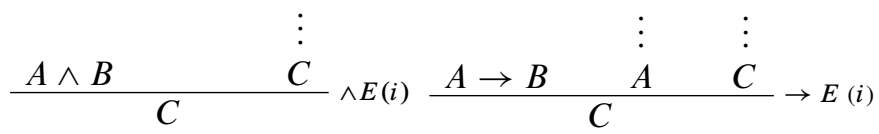

Remark 1.2 The usual (i.e., nonparallelized) rules $\wedge E$ and $\rightarrow E$ of natural deduction are trivially derivable in IR by replacing $C$ with either $A$ or $B$ in the former and with $B$ in the latter.

Last, the following liberalized rule $\vee E_{i r}$,

$$
\begin{aligned}
& \frac{\bar{A}}{\square(i)} \quad \bar{B} \square(i)
\end{aligned}
$$

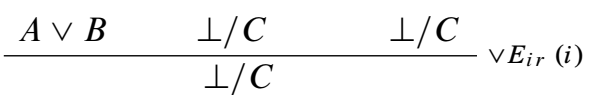

was invented by Tennant to allow in IR the proof of disjunctive syllogism; that is,

$$
(A \vee B), \neg A \vdash B,
$$

without making use of the intuitionistic rule $\perp E$.

Tennant explains this rule as follows: 
By stating it [rule $\vee E_{i r}$ ] graphically as we have, we are providing for the possibility that one of the case assumptions might lead to absurdity $(\perp)$. We are then permitted to bring down as the main conclusion whatever is concluded from the other case assumption.... Liberalizing proof by cases in this way is entirely natural, given how we reason informally. Suppose one is told that $A \vee B$ holds, along with certain other assumptions $X$, and one is required to prove that $C$ follows from the combined assumptions $X, A \vee B$. If one assumes $A$ and discovers that it is inconsistent with $X$, one simply stops one's investigation of that case, and turns to the case $B$. If $C$ follows in the latter case, one concludes $C$ as required. One does not go back to the conclusion of absurdity in the first case, and artificially dress it up with an application of the absurdity rule so as to make it also "yield" the conclusion $C$. ([7, p. 714]; italics in the original)

Our paper focuses only on natural deduction for IR and does not deal with the corresponding sequent calculus for IR. But, to conclude this section, I stress on the treatment of the deduction theorem and its converse. About this basic theorem, Tennant says the following:

The orthodox Deduction Theorem for logical calculi states that:

$$
X \vdash B \text { only if } X \backslash\{A\} \vdash A \rightarrow B \text {; and } X \vdash A \rightarrow B \text { only if } X, A \vdash B
$$

In IR, the first implication holds. But the converse fails, for in IR we have $\neg A \vdash A \rightarrow B$ but not: $\neg A, A \vdash B$. The lesson here is that we have to be careful to distinguish between asserting a conditional and making an inference. We have determined a conditional that is exactly what is needed in order to have the best possible system of relevant inference. The price of 'unpacking' a conditional is eternal vigilance with regard to the joint consistency of the new set of assumptions in play, and with regard to the possible logical truth of the new conclusion. [5, p. 344]

\section{IR Changes the Meaning of Disjunction Elimination}

Statement 2.1 It is provable that rule $\vee E_{i r}$ changes the established meaning of $\checkmark$ when $\vee$ is on the left of the turnstile.

\section{Proof}

$$
A \vee B \vdash C
$$

means in minimal logic that $C$ is a syntactical consequence of both $A$ and $B$; that is, (2) is provable if and only if it is provable that $A \vdash_{m} C$ and if it is provable that $B \vdash_{m} C$, whatever $A, B$, and $C$ are. In IR, the meaning of (2) changes if either $A$ or $B$ is equivalent to $\perp$, because via rule $\vee E_{i r}$,

$$
\perp \vee B \vdash B,
$$

but only because of the axiom $B \vdash B$ and in spite of the claim that $\perp \nvdash B$ in IR. Therefore, IR changes the meaning of $\vee$ when $\vee$ is on the left of the turnstile.

\section{The Law of Substitution of Logical Equivalents Fails in IR}

\section{Theorem 3.1 The law of substitution of logical equivalents fails in IR.}

Proof The logical equivalence in IR between $(\perp \vee B)$ and $B$ is provable:

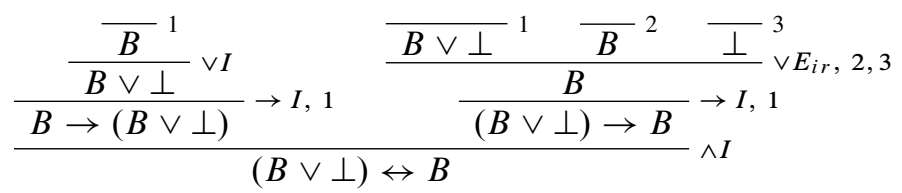


Nevertheless, $(\perp \vee B)$ and $B$ are not always substitutable salva veritate in IR. Indeed,

$$
\perp \vdash \perp \vee B
$$

is provable in IR (via rule $\vee I$ ), but if one replaces $\perp \vee B$ with $B$, one gets

$$
\perp \vdash B
$$

in contradiction to the claim of "relevance at the level of the turnstile" in IR (i.e., $\perp \nvdash B$ ). Therefore, the law of substitution of logical equivalents fails in IR because it is not true in IR that, in any context $\Gamma$,

$$
\Gamma \vdash \perp \vee B
$$

if and only if

$$
\Gamma \vdash B \text {. }
$$

\section{A Heavy Rule of Absurdity in IR}

IR does not differ from minimal logic only by rejecting the weakening rule. Contrary to minimal logic and in agreement with intuitionistic logic, $\perp$ is in IR an inference marker occurring with contradiction and being equivalent to any contradiction; but this difference with minimal logic entails some problematic theorems, such as the following.

Theorem 4.1 Unlike minimal logic, any contradiction is logically equivalent to any other one in IR; that is,

$$
\vdash(\neg A \wedge A) \leftrightarrow(\neg B \wedge B) .
$$

Proof

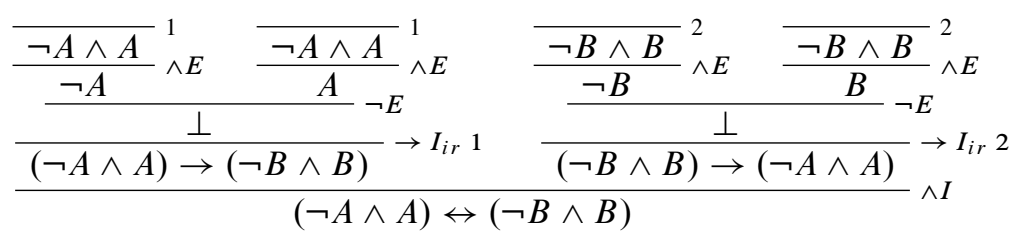

Theorem 4.1 leads to a way of avoiding in IR the relevance at the level of the turnstile.

Theorem 4.2 In IR, $B$ is deducible "at the level of the turnstile" from the formulas $\{(\neg A \wedge A) \leftrightarrow(\neg B \wedge B), \neg A, A\}$; that is,

$$
(\neg A \wedge A) \leftrightarrow(\neg B \wedge B), \neg A, A \vdash B
$$

is provable in IR via a proof in normal form.

\section{Proof}

$$
\frac{\frac{(\neg A \wedge A) \leftrightarrow(\neg B \wedge B)}{(\neg A \wedge A) \rightarrow(\neg B \wedge B)} \wedge E \quad \frac{\neg A \quad A}{\neg A \wedge A} \wedge I}{\frac{\neg B \wedge B}{B} \wedge E} \rightarrow E
$$

Remark 4.3 This proof is a proof in IR: it is in normal form, and the usual rules $\wedge E$ and $\rightarrow E$ are rules of IR because they are derivable in IR (Remark 1.2). 
Remark 4.4 Sequent (9) is semantically reducible to

$$
(\perp \leftrightarrow \perp), \perp \vdash B
$$

and therefore to

$$
\top, \perp \vdash B
$$

and last (via the left rule of conjunction introduction or, more directly, via the left rule of $\top$ elimination), this sequent is reducible to

$$
\perp \vdash B \text {. }
$$

\section{Conclusion}

The arguments of Sections 2, 3, and 4 provide three sufficient reasons to deny that IR is a core logic.

1. We expect that a core logic respects the univocal meaning of logical rules. It is not the case with IR: rule $\vee E_{i r}$ has not the same meaning when it is applied to a sequent like

$$
A \vee \neg \neg \neg \neg A \vdash \neg \neg A
$$

and when it is applied to

$$
(\neg A \wedge A) \vee B \vdash B .
$$

Sequent (13) is valid in IR because two sequents are provable (i.e., $A \vdash \neg \neg A$ and $\neg \neg \neg \neg A \vdash \neg \neg A$ ), but sequent (14) is valid in IR only because $B \vdash B$ is an axiom. (Of course, $\neg A \wedge A \vdash B$ is claimed to be unprovable in IR.)

2. At least since Leibniz, this law of substitution of logical equivalents is regarded as a basic logical law. To quote Leibniz's example (see [2, pp. 85-87]), if $A$ and $B$ have the same meaning, for example, "trilateral plane figure" and "plane figure triangular," then $A$ can be replaced with $B$ salva veritate in all statements (provided a context as referentially transparent as Euclidean geometry). Conversely, it is generally accepted in mathematical $\operatorname{logic}$ that, if $A$ cannot be replaced by $B$ in system $\mathbf{S}$, it is because $A$ and $B$ have different meanings; that is, they are not logically equivalent in $\mathbf{S}$. For example, $\neg \neg A$ cannot be replaced by $A$ salva veritate in intuitionistic logic, and $\perp \vee B$ cannot be replaced by $B$ in minimal logic. This law is essential to our understanding of the rules of logical systems in general. By proving that Leibniz's law fails in IR, Section 3 helps explain why this so-called core logic is so difficult to understand. Of course, Tennant could reply that the virtue of IR is to prevent the transitivity of deductions from sequents $\Gamma \vdash \perp \vee B$ to $\Gamma \vdash B$ when $\Gamma$ is itself $\perp$. But the trouble is that the logical equivalence between $\perp \vee B$ and $B$ is syntactically provable in IR regardless of context and therefore should be semantically valid in IR, also regardless of context; proof has been given in this paper that it is not the case in IR, and it is a serious problem if harmony between syntax and semantics is expected in core logic.

3. To my knowledge, nowhere has Tennant clearly explained the difference between a provable inference "at the level of the turnstile" and a provable conditional (i.e., an implication). After all, why should (ECQ) be unprovable, while the conditional $(\neg A \wedge A) \rightarrow B$ is a theorem of IR? This lack of harmony between provable sequents and provable conditionals is shocking. The 
proof given in Section 4 shows that Tennant's slogan is not even in harmony with the theorem of $\mathbf{I R}$, according to which any contradiction $(\neg A \wedge A)$ is equivalent to another one, say $(\neg B \wedge B)$. From this point of view, minimal logic is a better candidate to be a core logic. With minimal logic, by contrast with IR, you are not embarrassed to recognize that if you assume that any contradiction is equivalent to any other one, it is easy to show, at the level of the turnstile, that from this assumption and the assumption of $\neg A \wedge A$, you can infer $B$ (i.e., (9) is provable in minimal logic). In my opinion, it is contrary to the meaning of "core logic" to contradict minimal logic, and that is probably the main reason why Tennant's logic cannot be a core logic.

\section{Notes}

1. Tennant calls this rule "Dilution." I use the most usual name for this rule.

2. In [4, p. 189], Tennant repeats that IR "requires non-vacuous discharge of assumptions," but he adds in footnote 6 on the same page, "except, crucially, in one half of the rule of implication introduction!" This means that it is only for the other half of implication introduction (i.e., for rule $\rightarrow I_{i r}$ ) that vacuous discharge (i.e., weakening) is forbidden.

\section{References}

[1] David, R., K. Nour, and C. Raffalli, Introduction à la logique (Théorie de la démonstration, cours et exercices corrigés), Dunod, Paris, 2003. 3

[2] Leibniz, G. W., Recherches générales sur l'analyse des notions et des vérités: 24 thèses métaphysiques et autres textes logiques et métaphysiques, edited by J.-B. Rauzy, Presses universitaires de France, Paris, France, 1998. 6

[3] Prawitz, D., Natural Deduction-A Proof Theoretical Study, 2nd ed., Dover, Mineola, N.Y., 2006. MR 0193005. 2

[4] Tennant, N., Autologic, Edinburgh University Press, Edinburgh, 1992. 2, 3, 7

[5] Tennant, N., The Taming of the True, Oxford University Press, New York, 1997. Zbl 0929.03001. MR 1469976. 4

[6] Tennant, N., "Ultimate Normal Forms for Parallelized Natural Deductions," Logic Journal of the IGPL, vol. 10 (2002), pp. 299-337. MR 1914463. DOI 10.1093/jigpal/ 10.3.299. 2

[7] Tennant, N., "Relevance in Reasoning," pp. 696-726 in The Oxford Handbook of Philosophy of Mathematics and Logic, edited by S. Shapiro, Oxford University Press, Oxford, 2005. 1, 2, 4

\section{Acknowledgments}

I thank Neil Tennant for his friendly correspondence and his patience in explaining the intricacies of IR. I also had very useful discussions on this subject with Sara Negri, Jan von Plato, Konstantine Arkoudas, Roy Dyckhoff, Dominique Larchey-Wendling, and Richard Zach. I thank each of them, especially Roy Dyckhoff, because I am indebted to him for Theorem 3.1. Finally, my warmest thanks to my good old friends, Henri Astier and Christian Desmier, who have both revised and improved my English in this paper. 
Département de philosophie Université de Lorraine

Archives Poincaré-CNRS-UMR 7117

91 Avenue de la Libération

BP 454-54001 Nancy CEDEX

France

joseph.vidal-rosset@univ-lorraine.fr 